\title{
Model-theoretic Nature of the Laws of Motion in Newton's Principia
}

\author{
Toshio IsHIGAKI*
}

It is a commonly accepted view that Newtonian mechanics presupposes inertial frames of reference where the laws of motion hold. In the present paper, however, I will show that Newton himself, who believed in absolute space, did not make use of absolute space or even so-called inertial frames in dealing with actual motions of actual bodies like satellites, planets, and others in Book III of the Principia, but the conception of reality of force was essential for him, and that, in terms of modern space-time theoreticians, the space-time Newton specified by real force was Maxwellian, not neo-Newtonian or Galilean. Hence, it will become evident that for Newton the laws of motion was not universal statements that can be verified or falsified, but the scheme that enables us to construct the real picture of the world, as the model-theoreticians of science view fundamental laws.

\section{Introduction}

Isaac Newton asserted the existence of absolute space and absolute time, hence the spatio-temporal world consisting of absolute space and absolute time has been called Newtonian space-time, and his first two laws of motion ${ }^{1}$ have been regarded as valid primarily in absolute space. But as Newton demonstrates in Corollary V to Axioms of the Principia ${ }^{2}$ (for Axioms, Corollaries, and Propositions of the Principia, see Appendix to the present paper), the laws are derivatively valid in spatial frames of reference that move uniformly along straight lines in absolute space. Let us call these frames, Newtonian inertial frames, and the laws of motion holding in them, Newtonian laws of motion.

It is an accepted view, however, that what Newtonian mechanics as a physical theory really needs is not absolute space but only a class of frames of reference where the laws equivalently hold. Hence, modern authors ${ }^{3}$ have formulated Newtonian mechanics in such a space-time that there is a preferred class of spatial frames of reference among which no distinctions are made. This space-time is called neoNewtonian or Galilean. We call frames of this class neo-Newtonian inertial frames, and the laws of motion holding equally in them, neo-Newtonian laws of motion. Of course, the class of neo-Newtonian inertial frames should be taken to be extensionally equal to that of Newtonian inertial frames.

* Hokkaido University, Sapporo, Japan 
Whether in Newtonian or neo-Newtonian inertial frames, it is for the resultant force composed of all real forces on each body that the laws of motion should hold. Hence, if we are able to specify Newtonian or neo-Newtonian inertial frames in relation to actual material bodies, we can determine the resultant real force on each body from its motion with respect to these inertial frames, and, conversely, if we know the resultant real force on each body, we can specify inertial frames in relation to its actual motion. But if we also take apparent forces into consideration, then the laws formally hold in any frame for the sum of the resultant real force and the apparent force due to the acceleration of the reference frame relative to the inertial ones. Thus, the question becomes: How can one distinguish the real from the apparent force, for the sum of which the laws are always formally valid, that is, how can one determine the real and total forces on actual bodies, or, how can one concretely specify inertial frames?

In the present paper I will critically examine Newton's inductive inferences from Phenomena to the law of universal gravitation (Secs. 2 to 4 ) and his deductive inferences from the latter (Sec.5) presented in Book III of the Principia, where actual, not imaginary, phenomena, like the motions of planets, satellites, pendulums and others, are dealt with; and will reveal that Newton himself logically relies on neither absolute space nor inertial frames (Newtonian or neo-Newtonian), but exclusively on the conception of reality of force, in dealing with these actual phenomena, and hence that for Newton himself the laws of motion are not universal statements whose truth or falsity can be empirically tested by checking the predictions derived from them, but rather function to determine the structure of space by finding those forces that with other elements satisfy the laws and are acceptable to be real. Hence, the role of the laws of motion will be shown to agree with the model-theoretic view of science.

\section{Satellites}

In this section I will examine Newton's demonstration of Proposition I of Book III, in which he derives two statements about the motions of satellites of Jupiter:

S1. The forces by which the circumjovial planets are continually drawn off from rectilinear motion, and retained in their proper orbits, tend to Jupiter's centre.

S2. [These forces] are inversely as the squares of the distances of the places of those planets from that centre.

In the explanatory paragraph he says: 
[S1] appears from Phen. I, and Prop. II or III, Book I;

[S2] from Phen. I, and Cor. VI, Prop. IV, of the same Book.

Newton does not explicitly state, however, all observational premises of the demonstrations in Phenomenon I, so we must refer also to the unstated premises of those theorems on which he bases his demonstrations. Let us look at these proofs closely one at a time.

\section{Demonstrations of S1}

\section{(1). Demonstration by Proposition II of Book I.}

In Phenomenon I, Newton describes the motions of Jupiter's satellites as he thinks "known from astronomical observations or agreed by all astronomers". The relevant part is as follows:

the circumjovial planets, by radii drawn to Jupiter's centre, describe areas proportional to the times of description.

Clearly, the motions of Jupiter's satellites are described with respect to the spatial frame of reference where Jupiter's center is at rest and that does not rotate relative to the stars, which we call Jupiter-Star frame. But, logically speaking, that the motions are described relative to Jupiter-Star frame does not imply that the laws of motion should be applied to these motions in that frame, while Newton did so.

Newtonian-neo-Newtonian as well-laws of motion imply that the reference frames in Proposition II of Book I are inertial. Therefore, that Newton cites this proposition in demonstration of Sl would mean that he regards Jupiter-Star frame as inertial. But is Jupiter-Star frame inertial ? Newton did not give any reason why he applied the laws in such a way. I shall consider this question below.

Newton presents another proof of S1.

(2). Demonstration by Proposition III of Book I.

Under Newtonian or neo-Newtonian laws of motion, Proposition III of Book I implies that Jupiter and its satellites are accelerated equally and parallel relative to inertial frames by some force, and, hence, that Jupiter-Star frame also has the same acceleration. Which, then, are the frames to be regarded as inertial in the demonstration? And what is the accelerating force? Though Newton himself does not explicitly state anything about this, we can easily conjecture that the frame to be regarded as at least approximately inertial is one, which we call Sun-Star frame, 
where the center of the sun is at rest and which does not rotate relative to stars, and that the accelerating force is gravity towards the sun.

If the first part of this conjecture is valid, i.e., Sun-Star frame is inertial, then we should be able to answer the question posed in (1). That is, looking from the inertial Sun-Star frame, one could regard Jupiter-Star frame as inertial for a sufficiently short time. Since the longest of the periods of the satellites about Jupiter is $16 \mathrm{~d} 16 \mathrm{~h} 32 \mathrm{~m} 9 \mathrm{~s}$ and Jupiter's period about the sun is $4332.514 \mathrm{~d}$, according to the tables in Book III, the condition for taking Jupiter-Star frame to be regarded approximately as inertial is satisfied. But, of course, Jupiter-Star frame itself is not inertial.

Even if we regard Sun-Star frame as inertial, however (this is a premise of the demonstration for motions of planets in the next proposition of Book III), the demonstration by Proposition III further requires the second part of our conjecture to be valid, i.e., that Jupiter and its satellites are attracted by and undergo the same acceleration towards the sun. This is a consequence of the law of universal gravitation that Newton intends to derive inductively from the Proposition I and others. Hence, his inductive inference from phenomena to universal gravitation appears to be open to the charge of petitio principii.

Our questions thus become: Is Sun-Star frame inertial, and, if so, does the demonstration by Proposition III assume the law of universal gravitation? I shall return to these problems in section 6 .

Let us continue to follow Newton's demonstrations, taking up that of S2.

\section{Demonstration of $\mathbf{S} 2$ by Corollary VI to Proposition IV of Book I.}

Newton gives only one demonstration of S2. The remaining part of Phenomenon $I$ is as follows :

[The circumjovial planets'] periodic times, the fixed stars being at rest, are as the $3 / 2$ th power of their distances from [Jupiter's] centre.

But it is impossible to derive $\mathrm{S} 2$ only from phenomena explicitly stated there. In the explanatory paragraph Newton further states:

The orbits of these planets differ but insensibly from circles concentric to Jupiter.

From these phenomena he succeeds in demonstrating S2 by applying the laws of motion in Jupiter-Star frame. The same problems as in S1, however, arise by Corollaries V and VI of Laws of Motion, though Newton says nothing about them. 


\section{Planets}

As regards planets, Newton presents three Phenomena. However, the phenomena that he actually assumes in determination of force on planets are not restricted to these. Accordingly, as in Sec. 2, I will examine the premises of the theorems and corollaries that he uses in his demonstrations.

In Proposition II of Book III he derives two statements as in the case of satellites :

P1. The forces by which the primary planets are ... retained in their proper orbits, tend to the sun;

P2. [these forces] are inversely as the squares of the distances ... from the sun's centre.

$\mathrm{Pl}$ is derived, by applying the laws of motion in Sun-Star frame, from Phenomenon $\mathrm{V}$ :

the areas which [the primary planets] describe by radii drawn to the sun are proportional to the times of description.

In this demonstration, however, Newton refers only to Proposition II, not to Proposition III of Book I. Thus, under Newtonian, and neo-Newtonian, laws of motion, only one question seems to arise: Is Sun-Star frame inertial ?

But Newton refers to another possible reference frame in the same Phenomenon $\mathrm{V}$, namely the frame where the center of the earth is at rest and that does not rotate relative to the stars, which we call Earth-Star frame:

The primary planets, by radii drawn to the earth, describe areas in no wise proportional to the times.

The two statements just cited correspond to the passage in Phenomenon IV :

whether of the sun about the earth, or of the earth about the sun.

The reason Newton adopts Sun-Star rather than Earth-Star frame is evident. One could not obtain any force on planets that tends to physical bodies when applying the laws in Earth-Star frame, but could obtain a force on them that tends to the sun (a physical body) in Sun-Star frame. Newton chose Sun-Star frame as a reference frame for the laws of motion because a force that he could consider physically meaningful was obtained by such an application of the laws. This 
supposition about his reasoning is supported by the following statement in The System of the World (Sec. 4) :

That forces should be directed to no body on which they physically depend,..., is a hypothesis too incongruous.

Therefore, if Newton regarded Sun-Star frame as inertial, it is because, so to speak, real force on planets would appear by applying the laws of motion in that frame.

But can the inertiality of the reference frame be implied by the fact that real force appears by applying the laws of motion in that frame? Of course not. For, as Newton himself states (Sec. 8, The System):

it may be alleged that the sun and planets are impelled by some other force equally and in the direction of parallel lines; but by such a force ... no change would happen in the situation of the planets one to another.

The inertiality of the reference frame would require that other real forces, if any, on bodies were null in sum, than the forces that appear by the application of the laws in the frame. How, then, can one ascertain this? I shall return to this problem in Sec. 6 .

The premises for the derivation of P2 are not entirely presented in Phenomena III to V. But, since Newton gives two demonstrations, by Corollary VI to Proposition IV and by Corollary I to Proposition XLV of Book I, we can easily state these premises.

\section{Demonstration by Corollary VI to Proposition IV.}

The premises of Corollary VI are, as in the case of 2 in Sec. 2, that the primary planets move in circles concentric to the sun and that their periods are as the $3 / 2$ power of the semidiameters of their orbits in Sun-Star frame. Only the latter part is explicitly stated in Phenomenon IV, while 'their mean distances from the sun' is used there instead of 'the semidiameters of their orbits.' It is evident that the laws of motion are applied in Sun-Star frame, i.e., the frame where phenomena are given.

While the demonstration of the centripetal force in Proposition IV of Book I refers only to Proposition II but not to Proposition III of the Book, therefore, the same problems will arise as in the above case of $\mathrm{P} 1$.

Let us now see another demonstration of P2.

\section{Demonstration by Corollary I to Proposition XLV.}

The demonstration is briefly as follows: 
Consider a body $P$ moving along an ellipse $E$ with a constant areal velocity with respect to one of its focuses $F$ in a plane $S_{1}$ to which $E$ is fixed. $E$, hence $S_{1}$, rotates, while being contained in another plane $S_{2}$, about $F$ fixed at the same time to $S_{2}$, such that $P$ moves with another constant areal velocity with respect to $F$ in $S_{2}$ also. Therefore, $P$ moves only by respective centripetal forces to $F$, seen in $S_{1}$ and in $S_{2}$. The centripetal force in $S_{1}$ is proved to be in inverse proportion to the

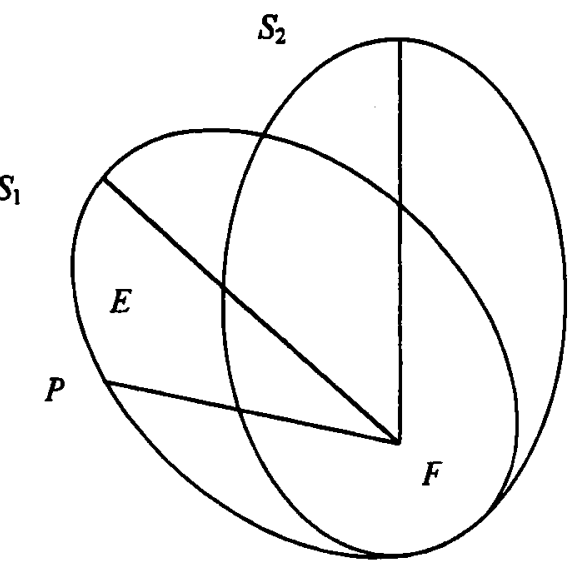
square of the distance between $P$ and $F$, but that in $S_{2}$ is not.

Newton proves (Prop. XLIV) that, when seen in $S_{2}$, the centripetal force on $P$ towards $F$ is the sum of the force being in inverse proportion to the square of the distance between $P$ and $F$, and the force being in inverse proportion to the cube of that distance, and further gives (Prop. XLV) to the sum of these two forces an expression in terms of the power of the distance, under the assumption that the ellipse $E$ is very nearly to a circle.

Now, Corollary I to Proposition XLV is thus:

if the whole angular motion, with which the body returns to the same apse, be to the angular motion of one revolution, or $360^{\circ}$, as any number as $m$ to another as $n$, and the altitude [i.e. distance $P F$ ] be called $\mathrm{A}$; the force will be as the power $\mathbf{A}^{(n n / m m)-3}$ of the altitude $\mathbf{A}$.

By this corollary Newton derives P2 from the quiescence of the aphelion points (i.e., $n / m=1$ ) in Sun-Star frame.

Therefore, his demonstration of the inverse square law of forces on planets from the quiescence of the aphelion points means that he assumes the orbits of the planets to be ellipses fixed to Sun-Star frame. Again we are led to the question: Is Sun-Star frame inertial ?

Newton does not present any reason for the inertiality of Sun-Star frame in these two demonstrations. Indeed, and to the contrary, he says (I have already cited a part of this) :

It may be alleged that the sun and planets are impelled by some other force equally and in the direction of parallel lines; but by such a force (by Cor. VI 
of the Laws of Motion) no change would happen in the situation of the planets one to another, nor any sensible effect follow: but our business is with the causes of sensible effect.

This means that Newton admits the possibility of linearly accelerating movement of Sun-Star frame. Hence, the situation is just the same as for the satellites of Jupiter, except that one could specify neither the cause of the other force nor any possibly inertial frame. Accordingly, we might have to take into consideration Proposition III of Book I in the case of planets also.

In a later section of The System (Sec. 27), however, Newton abruptly tell us :

Reject that translation of the whole system, and the sun will be almost quiescent in the centre thereof,

without giving any reason. So, in Sec. 6 I shall study consequences of the possibility that Newton admitted once.

\section{The moon}

Newton's treatment of the moon is rather complicated. He states in Phenomenon VI :

That the moon, by a radius drawn to the earth's centre, describes an area proportional to the time of description.

Although there is no indication of the reference frame for the phenomenon in this statement, it is evident that the description of the motion of the moon is given relative to Earth-Star frame.

Newton applies the laws to the motion of the moon in Earth-Star frame without giving any reason for doing so, and demonstrates (Prop. III) that:

M1. The force by which the moon is retained in her orbit tends to the centre of the earth.

M2. [That force] is inversely as the square of the distance of its place from the earth's centre.

As to M1, Newton gives "Proposition II or III of Book I" as bases for its demonstration. Hence, there arise the same problems as in the case of the satellites of Jupiter.

For M2, Newton's inference is as follows: 
First, he mentions the slow motion of the moon's apogee in Earth-Star frame. He represents the motion of the moon, as in 2.2 of Sec. 3 above, as movement along an ellipse revolving about one of its focuses. And, from the motion of the moon's apogee amounting to $3^{\circ} 3^{\prime}$ he derives by Cor. I, Prop. XLV, Book I, that the force is in inverse proportion to $\mathrm{D}^{2+(4 / 243)}$, where $\mathrm{D}$ is the distance of the moon from the earth's center. Then, about the motion of the apogee, i.e., about $4 / 243$ in the exponent, he states that:

this increase [of the exponent] is due to the action of the sun,

and, neglecting it, he derives M2.

Hence, we are faced with the problem of the action of the sun at this stage of the reasoning, in addition to the problems of reference frames.

Let us see the implications of the neglect of the motion of the apogee. Since Newton refers to Cor. I, Prop. XLV, Book I (see 2 in Sec. 3 above), he assumes the orbit of the moon to be a fixed ellipse in Earth-Star frame, in addition to Phenomenon VI. Further, from the reason for the neglect that he mentions, it is evident that he accepts not only the earth but the moon as attracted by the sun (the sun's attraction on the earth could be taken as proved in Proposition II, but the sun's attraction on the moon is derivable only from the law of universal gravitation that is to be demonstrated at a later stage of his reasoning), and he uses the solution of a so-called restricted three-body problem of the sun, the earth, and the moon.

Therefore, Newton seems to be open to the charge of petitio principii again, since, here, he compares gravity of terrestrial bodies with the force on the moon towards the earth that obeys the inverse square law (Prop. IV) ; and then generalizes gravity to celestial bodies (Prop. V), and uses the generalized gravity to derive the inverse square law of the force on the moon towards the earth.

In The System (sec. 10) he presents another demonstration of M2. That is, he simply assumes that the moon moves uniformly in a circle with its center at the center of the earth in Earth-Star frame, and that the centripetal force on the moon towards the earth is in inverse proportion to the square of the distance from the earth. Then he calculates the acceleration near the surface of the earth by the supposed centripetal force under these assumptions, and finds it is approximately equal to the acceleration of terrestrial bodies by gravity. $\mathrm{He}$ thus identifies the centripetal force on the moon with gravity, and at the same time concludes the supposition about the force to be ascertained:

The circumterrestrial force decreases inversely as the square of the distances from the earth. 
While in this hypothetico-deductive reasoning itself the law of universal gravitation is not used, questions will be raised in just the same way as the case of Jupiter's satellites (see the end of 1 in Sec. 2).

In Sec. 6 I shall attempt to interpret his demonstrations independently of the inertiality of the reference frames.

\section{Solar system}

In the preceding sections I stated that some of Newton's inductive inferences seem to assume the law of universal gravitation. In the present section I will examine his demonstrations about motions of bodies in the solar system from the law of universal gravitation.

In Proposition XI Newton asserts :

That the common centre of gravity of the earth, the sun, and all the planets, is immovable.

In the derivation of this statement, he uses Corollary IV of Laws to demonstrate that the center is either at rest or moves uniformly in a straight line. The premise of this corollary is that bodies in question act upon each other and that no other action is exerted on them. Hence, it is evident that Newton has considered universal gravitation only within the solar system. Let us call the law of universal gravitation confined in this way the confined law of universal gravitation. What are the reasons for such a use of the law? More specifically, why is it unnecessary to take into consideration possible forces from outside the solar system, e.g., according to the law of universal gravitation?

In any case Newton derives, using the confined law of universal gravitation, that the center of gravity of the solar system is either at rest or moves uniformly in a straight line. But relative to what frame "is it at rest or does it move uniformly in a straight line?" Probably relative to absolute space. If so, Proposition XI means that the center of gravity is immovable in one of Newtonian inertial frames. Then, he uses only one Hypothesis I :

the centre of the system of the world is immovable,

to conclude that the center of gravity of the solar system is at rest in absolute space, whether the earth or the sun occupies the center of the system of the world (Prop. $\mathrm{XI}$ ). Of course, he could not give any observational or experimental support for this hypothesis. How, then, can one empirically determine the reference frame?

From the confined law of universal gravitation and relative motions of bodies 
in the solar system, it can be derived that the sun approximately occupies the center of gravity of the solar system (see 2 in Sec. 6).$^{5}$ Hence, one can determine at least approximately the center of gravity of the solar system in relation to actual physical bodies. Newton asserts, by identifying the center of the system of the world with the center of gravity of the solar system, that the sun is approximately at rest in absolute space. So far, however, one can only determine one point of absolute space in relation to actual bodies by the Hypothesis, not the entirety of absolute space.

The problems of motions of the sun, the earth, other planets, and satellites in the solar system are so-called many-body problems. Newton analyses these problems in terms of two-body or restricted three-body problems, and tries to solve approximately the originals by combining the solutions of these manageable problems. So he demonstrates Proposition XIV:

The aphelions and nodes of the orbits of the planets are fixed.

Thus he has been able to determine completely the absolute frame in relation to relative motions of actual bodies (i.e., to the aphelions and nodes), that is, the absolute frame is one where the sun and the aphelions and nodes of planets' orbits are at rest. He, then, asserts in Corollary I :

The fixed stars are immovable, seeing they keep the same position to the aphelions and nodes of the planets.

That is, the fixed stars are immovable in absolute space.

Following Newton's demonstrations from the law of universal gravitation, we can say that what he has assumed about absolute space is merely the resting of the center of gravity of the solar system in absolute space. It is not a premise but a consequence for him that the fixed stars are immovable in absolute space. His assumption, indeed, gives a basis for the confined law of universal gravitation, since, if forces that act on the solar system from outside are not null in sum, its center of gravity could not be at rest in absolute space.

As Newton could not provide any observational or theoretical support for his assumption, however, one cannot specify the absolute frame without relying on a mere assumption. We next consider how the situation should be interpreted under neo-Newtonian laws of motion that do not presuppose absolute space.

Is it evident under neo-Newtonian laws of motion that the center of gravity is at rest in one of the inertial frames? To answer that question affirmatively would require that the sum of all forces on the solar system from outside be null. Is there any evidence to support this as valid? As cited above, Newton himself admitted once : 
The sun and planets [might be] impelled by some other force equally and in the direction of parallel lines.

Logically speaking, Newton is right. The center of gravity of the solar system might be accelerated. Hence, the reference frame where the center of gravity and the aphelions and nodes are asserted to be at rest, which we call Solar frame, might be accelerated, that is, not be inertial. Nevertheless, one could successfully apply the laws of motion to relative motions of bodies in the solar system by using the confined law of universal gravitation, and obtain Solar frame as a frame where the application of the laws works well. That is, Newton successfully uses the laws of motion for the relative motions of the sun and planets, and for the confined law of universal gravitation, to determine an adequate reference frame in relation to those motions.

As for the premises of this inference, those relative motions, and some of their observational ingredients, are given independently of the absolute and inertial frames, and the law of universal gravitation confined to within the solar system is already accepted at this stage of his reasoning as real, and concretely specified with relative positions and masses of the bodies in the solar system. These premises require neither absolute space nor even inertial frames. And the laws of motion are applied in a possibly accelerating frame. (Remember that we are considering this without assuming Newton's Hypothesis I.)

Newton does not rely on stars to decide the reference frame for the laws of motion here, unlike in the cases of satellites, planets, and the moon. We could summarize his reasoning about the solar system as follows, but without Hypothesis I :

Starting from concretely specifiable forces on the bodies in question (i.e., gravity) and their observationally given relative motions, he has succeeded in determining a reference frame for the laws of motion in relation to their observationally specified locations.

Here, neither Newtonian nor neo-Newtonian laws of motion are used. The attempt is to determine, in relation to actual bodies, those reference frames where, so to speak, schematic laws of motion hold for observational relative motions and for specified forces to be regarded as real.

\section{Conclusion}

In Secs. 2 to 4 we saw that the motions of satellites, planets, and the moon may not be explicable in Newtonian or neo-Newtonian space-time until Sun-Star frame 
is regarded as inertial. But, in Sec. 5, we saw also that there is no evidence for Sun-Star frame being inertial and that Newton himself does not rely on inertial frames in his treatment of motions of bodies in the solar system, except for his mere Hypothesis about the center of the system of the world. Hence, it is not under the Newtonian or neo-Newtonian interpretation of the laws of motion that the treatment of motions in the inductive reasonings could obtain legitimacy. How, then, can we justify those inductive reasonings?

In Sec. 5 we concluded that the laws of motion are applied to observational relative motions and specifiable real forces, and that the reference frames are thereby determined in relation to observations. Let us follow this line to reinterpret the treatments of satellites and planets.

\section{Satellites of Jupiter.}

The satellites move uniformly in circles concentric to Jupiter in Jupiter-Star frame such that their periods are as the $3 / 2$ power of their distances from Jupiter's center. When one considers only the relative motions of Jupiter and its satellites, and if one chooses reference frames other than Jupiter-Star frame or frames uniformly translating relative to it, then one cannot obtain seemingly real forces on the satellites by applying the laws of motion in these other frames, for the application of the laws in Jupiter-Star frame produces the force towards Jupiter that obeys the inverse square law of distance from it, whereas applications of the laws in those other frames would give quite complex and strange forces on satellites that might not tend towards any physical body. Hence, if one requires that applications of the laws of motion to relative motions should produce forces capable of being regarded as real, one would almost inevitably use Jupiter-Star frame.

If one considers the relative motions of the sun, Jupiter, and its satellites, however, one would have to adopt Sun-Star frame as a reference frame, where Jupiter and its satellites would be acted upon by some, not yet specifiable, force towards the sun and, in addition, the satellites would be acted upon by a force towards Jupiter that is in inverse proportion to the square of the distance from Jupiter. ${ }^{6}$

In both cases the forces to appear tend to physical bodies.

\section{Planets.}

As in the first case of 1 above, if one adopts frames other than Sun-Star frame or frames uniformly translating relative to it, one could not derive seemingly real forces on planets from the premises about their motions in Sec. 2. But the case corresponding to the second of 1 above does not arise because the possible forces on the solar system as a whole could not concretely be specified. (See the end of the present section.) 
Therefore, taking the conclusions of Sec. 5 into consideration, we can conclude that Newton might use his laws of motion in either of the following two ways, perhaps except for the case of the moon.

(1) When forces, but possibly not all, on the bodies in question are concretely specified and regarded as real, he attempts to determine reference frames in relation to the relative locations of the bodies in question, such that the laws of motion hold for their relative motions and for those forces on the bodies.

(2) When such forces are not given, he attempts to determine reference frames in relation to observations such that those forces on the bodies that would be determined by applications of the laws of motion in the frames could be regarded as real, but possibly not all.

In both cases what is essential is reality of force. Newton neither identifies any inertial frame in relation to actual bodies, nor ascertains the totality of forces on bodies, when applying the laws of motion. From (2) we can understand well the fact that Newton refers to alternative frames in Phenomenon $\mathrm{V}$ and then chooses one, i.e., Sun-Star frame.

About reality of force, Newton states in The System (Sec. 4) to the effect that:

(3) Real forces physically depend on some body.

(4) Real forces decrease in receding from the fountain from which they flow.

Of course, these criteria are not enough to distinguish real from apparent forces. But it appears certain that real force is fundamental for him in dealing with the actual motions of actual bodies, not absolute or inertial frames.

My interpretation of his demonstrations agrees with his statement (Scholium to Definitions of the Principia) :

We have some arguments to guide us, partly from the apparent [i.e., relative] motions, ... partly from the forces.

Lastly, let us turn to the exceptional case of the moon.

\section{The Moon.}

Proposition III of Book III in the Principia would not be derived as a part of the inductive inferences, since it presupposes at least approximate solutions to the three-body problem of the sun, the earth, and the moon, under the law of universal 
gravitation, which implies the inverse square law of force on the moon towards the earth. Hence, this treatment of the moon's motion should be taken to be a deductive explication by universal gravitation, rather than a part of the inductive inferences from phenomena to universal gravitation.

The other demonstration in The System cited in Sec. 4, however, may allow us to interpret it as a part of the induction. If one identifies, from the beginning, the force of gravity on terrestrial bodies near the surface of the earth with some supposed force on the moon, then one could not but adopt Earth-Star or Sun-Star frame to obtain seemingly real forces as in the case of the satellites of Jupiter (see 1 above). And this interpretation would agree with the second way of applying the laws of motion.

Now we can see the model-theoretic nature of the laws of motion in Book III of Newton's Principia. We have seen that Newton does not use any hypotheses about which frame of space should be absolute or inertial when dealing with actual motions of actual material bodies. Hence the laws of motion are not universal, testable or falsifiable, statements for him, because, if so, he must have derived observational predictions from those laws with some initial states of bodies, which would require empirical specification of absolute frame or, at least, inertial frames, and the total real force on each material body, which is impossible. The laws of motion work for him as Axioms in the modern sense that enable him to construct their models out of presumable components of our world, which might give us some support to model-theoretic view of science. Since the most fundamental frame that he could specify by the laws of motion was Sun-Star or Solar one and this might be possibly accelerated linearly, the space-time he specified was neither Newtonian nor neo-Newtonian but Maxwellian.?

In the history of Newtonian mechanics, while Newton and others succeeded in applying the laws of motion to motions of bodies of the solar system in Solar frame, the frame has never been confirmed to be inertial, and it has been accepted from the beginning of this century that the solar system itself moves circularly about some point in the universe (i.e., the galactic rotation), as Thomas Wright, Lambert, Kant, and $\mathrm{Herschel}^{8}$ anticipated as early as the eighteenth century.

\section{Appendix}

Extracts from Principia.

Law 1.

Every body continues in its state of rest, or of uniform motion in a right line, 
unless it is compelled to change that state by forces impressed upon it.

Law 2.

The change of motion is proportional to the motive force impressed; and is made in the direction of the right line in which that force is impressed.

\section{Corollary IV.}

The common centre of gravity of two or more bodies does not alter its state of motion or rest by the actions of the bodies among themselves; and therefore the common centre of gravity of all bodies acting upon each other (excluding external actions and impediments) is either at rest, or moves uniformly in a right line.

\section{Corollary V.}

The motions of bodies included in a given space are the same among themselves, whether that space is at rest, or moves uniformly forwards in a right line without any circular motion.

\section{Corollary VI.}

If bodies, moved in any manner among themselves, are urged in the direction of parallel lines by equal accelerative force, they will all continue to move among themselves, after the same manner as if they had not been urged by those forces.

\section{Proposition II.}

Every body that moves in any curved line described in a plane, and by a radius drawn to a point either immovable, or moving forwards with an uniform rectilinear motion, describes about that point areas proportional to the times, is urged by a centripetal force directed to that point.

\section{Proposition III.}

Every body, that by a radius drawn to the centre of another body, howsoever moved, describes areas about that centre proportional to the times, is urged by a force compounded of the centripetal force tending to that other body, and of all the accelerative force by which that other body is impelled.

\section{Notes}

1. The third law of motion shall not concern us here.

2. Isaac Newton, Sir Isaac Newton's Mathematical Principles of Natural Philosophy and His System of the World, translated by A. Motte and revised by F. Cajori (University of California Press, Berkeley and Los Angeles, Cal., 1966).

3. John Earman, World Enough And Space-Time (MIT Press, Cambridge, Mass., 1989) ; 
Michael Friedman, Foundations of Space-Time Theories (Princeton Univ. Press, Princeton, N.J., 1983).

4. Newton calculates the centrifugal force due to the rotation of the earth in Earth-Star frame in the Vellum Manuscript (John Herivel, The Background to Newton's Principia (Oxford University Press, Oxford, 1965), III), and in Proposition XIX, Book III of the Principia.

5. Since the relative motions of planets and the sun are approximately represented in Sun-Star frame under the inverse square law of force on planets towards the sun, one must take the mass of the sun to be immensely larger than those of planets in order to represent their motions under the law of universal gravitation.

6. Strictly speaking, there remains another possibility that the sun and the satellites of Jupiter revolve about Jupiter in Jupiter-Star frame so that the forces that retain them in their orbit about Jupiter tend to Jupiter's center. But when one takes another planet's motion into consideration, the force on this planet will tend no physical body.

7. Maxwellian space-time is one such that whether frames are rotating or non-rotating is meaningful but whether they are linearly accelerating or not is meaningless. See John Earman, World Enough And Space-Time (MIT Press, Cambridge, Mass., 1989), Ch. 2, Sec. 3.

8. Harlow Shapley and Helen E. Howarth, eds., $A$ Source Book in Astronomy (McGrawHill, N.Y., 1929), pp. 113-130. William Herschel, "On the Proper Motion of the Sun and Solar System", The Scientific Papers of Sir William Herschel (Royal Society and Royal Astronomical Society, London, 1912), pp. 108-30. 